Article

\title{
Genome-Wide Analysis of Glycoside Hydrolase Family $1 \beta$-glucosidase Genes in Brassica rapa and Their Potential Role in Pollen Development
}

\author{
Xiangshu Dong ${ }^{1, *(\mathbb{C}, \text {, Yuan Jiang }}{ }^{1}$ and Yoonkang Hur ${ }^{2, *}$ \\ 1 School of Agriculture, Yunnan University, Kunming 650091, China; jiangyuan@mail.ynu.edu.cn \\ 2 Department of Biological Sciences, Chungnam National University, Daejeon 34141, Korea \\ * Correspondence: dongxiangshu@ynu.edu.cn (X.D.); ykhur@cnu.ac.kr (Y.H.); \\ Tel.: +86-871-6503-1539 (X.D.); +82-42-821-6279 (Y.H.)
}

Received: 28 February 2019; Accepted: 1 April 2019; Published: 3 April 2019

\begin{abstract}
Glycoside hydrolase family 1 (GH1) $\beta$-glucosidases (BGLUs) are encoded by a large number of genes, and are involved in many developmental processes and stress responses in plants. Due to their importance in plant growth and development, genome-wide analyses have been conducted in model plants (Arabidopsis and rice) and maize, but not in Brassica species, which are important vegetable crops. In this study, we systematically analyzed $B$. rapa BGLUs (BrBGLUs), and demonstrated the involvement of several genes in pollen development. Sixty-four BrBGLUs were identified in Brassica databases, which were anchored onto 10 chromosomes, with 10 tandem duplications. Phylogenetic analysis revealed that 64 genes were classified into 10 subgroups, and each subgroup had relatively conserved intron/exon structures. Clustering with Arabidopsis BGLUs (AtBGLUs) facilitated the identification of several important subgroups for flavonoid metabolism, the production of glucosinolates, the regulation of abscisic acid (ABA) levels, and other defense-related compounds. At least six BrBGLUs might be involved in pollen development. The expression of BrBGLU10/AtBGLU20, the analysis of co-expressed genes, and the examination of knocked down Arabidopsis plants strongly suggests that BrBGLU10/AtBGLU20 has an indispensable function in pollen development. The results that are obtained from this study may provide valuable information for the further understanding of $\beta$-glucosidase function and Brassica breeding, for nutraceuticals-rich Brassica crops.
\end{abstract}

Keywords: $\beta$-glucosidase; Brassica rapa; BrBGLU10; pollen development; co-expression analysis

\section{Introduction}

Glycoside hydrolases (EC 3.2.1) are classified into a group of enzymes that hydrolyze the glycosidic bonds of carbohydrates [1]. At the end of March in 2019, 161 families have been identified and classified in the CAZy (Carbohydrate-Active enZYmes) database [2,3]. Among these families, the glycoside hydrolase $(\mathrm{GH})$ family 1 is recognized for its $\beta$-glycosidase activity, which largely contributes to various developmental processes and stress responses in plants [4,5]. Genome-wide analysis of GH1 $\beta$-glycosidase genes (BGLUs) has been conducted in three plant species: Arabidopsis, with 48 genes grouped into 10 subfamilies [6]; rice, with 40 genes grouped into eight subfamilies [5]; and maize, with 26 genes grouped into four subfamilies [7,8]. Recently, a comparison between the Arabidopsis and rice $B G L U$ s with respect to sequence identity and expression revealed that these exhibited substantial tissue specificity and differential responses to various stress treatments, although these have a high degree of similarity [9]. However, no systematic analysis of BGLUs in Brassica rapa, which is an important vegetable crop, has been performed to date. 
In addition to classifications based on genomic DNA organization, Arabidopsis BGLUs (AtBGLUs) could be classified in relation to their known functions, which shows that genes within the same subfamily may function in similar processes. A large number of $A t B G L U$ s are involved in flavonoid metabolism: AtBGLU1-6 for flavonol accumulation [10,11], AtBGLU7-11 for anthocyanin glucosyltransferase [11,12], and AtBGLU12-17 for flavonoid utilization [10,13]. Seven genes (AtBGLU26, AtBGLU34-39) function as myrosinases for chemical defense against herbivores and pathogen attacks [14-16]. AtBGLU18 and AtBGLU33 regulate ABA responses by increasing ABA levels through the hydrolysis of glucose-conjugated ABA (ABA-GE) $[17,18]$. Scopolin, which is specifically produced in the roots, and which plays a role in a defense against pathogen attack and abiotic stresses $[19,20]$, is controlled by ArBGLU21-23 [21,22]. The gene products encoded by AtBGLU45 and AtBGLU46 hydrolyze monolignol glucosides, thereby regulating lignin biosynthesis [23]. AtBGLU42 is involved in the induction of systemic resistance to bacterial disease, and the release of iron-mobilizing phenolic metabolites during iron deficiency [24]. However, no gene has been reported, with respect to pollen development.

During pollen development, the tapetum secretes various components, such as lipidic precursors and lipidics onto the pollen surface, leading to the formation of sculptured exine and exine cavities by hydrolyzation and other reactions [25]. In addition to lipid components, pollen wall development requires the regulation of polysaccharide metabolism [26], suggesting a possible involvement of the hydrolysis of glycosidic bonds of carbohydrates. Glycoside hydrolase has been reported involved in the cell wall polysaccharide degradation [27] and their coding genes were downregulated in the OsTDR (Tapetum Degeneration Retardation) mutant [28] and the sterile floral buds of B. rapa [29], indicating a possibility that $\beta$-glucosidase may play a role in pollen development.

In this study, we systematically identified Brassica rapa $\beta$-glycosidase genes (BrBGLUs) and analyzed their expression patterns and phylogenetic relationships. In addition, in silico analyses indicated that BrBGLU10/AtBGLU20 have conserved functions during pollen development, and knocking down AtBGLU20 using antisense oligos in Arabidopsis results in the production of aborted pollen grains. Furthermore, bioinformatics and molecular analyses provide valuable information on the function of BrBGLUs during pollen development.

\section{Results}

\subsection{Identification and Chromosomal Distribution of BrBGLUs}

After a HMM (Hidden Markov Model) search, 64 BrBGLU genes were identified and designated as BrBGLU1 to BrBGLU64, according to their positions on the chromosomes (Figure 1). The locus ID, genome location, coding sequence (CDS) length, and the protein length of the BrBGLUs are listed in Table 1. The genomic DNA sequences of the BrBGLUs ranged from $390 \mathrm{bp}$ to $9617 \mathrm{bp}$. While the average length was $1293 \mathrm{bp}$, the length of the CDS of the BrBGLUs ranged from $267 \mathrm{bp}$ to 2058 bp. The BrBGLU genes were heterogeneously distributed among all 10 chromosomes of B. rapa. Chromosome 5 contained the largest number of BrBGLU genes, comprising 15 members $(23.4 \%)$, whereas chromosome 2 contained only one gene member. We also detected tandem arrays of the $B r B G L U$ genes among the $10 \mathrm{~B}$. rapa chromosomes. The tandem array was defined as 'multiple BrBGLU genes located in neighboring or the same intergenic region' [30]. Ten BrBGLU gene clusters were found on chromosomes A01, A03, A05, A07, and A09. Chromosome 5 contained the maximum number of clusters, comprising $11 \mathrm{BrBGLUs.}$ 
Table 1. Characteristics of the GH1 (Glycoside hydrolase family 1) gene family in Brassica rapa.

\begin{tabular}{|c|c|c|c|c|c|c|c|c|c|c|c|}
\hline \multirow{2}{*}{ Locus ID } & \multirow{2}{*}{ Gene Name } & \multirow{2}{*}{$\begin{array}{l}\text { CDS Length } \\
\text { (bp) }\end{array}$} & \multirow{2}{*}{$\begin{array}{c}\text { Protein } \\
\text { Length (aa) }\end{array}$} & \multirow{2}{*}{ Chromosome } & \multirow{2}{*}{ Gene Start } & \multirow{2}{*}{ Gene End } & \multirow{2}{*}{$\begin{array}{c}\text { gDNA } \\
\text { Length (bp) }\end{array}$} & \multirow{2}{*}{$\begin{array}{l}\text { No. of } \\
\text { Exons }\end{array}$} & \multicolumn{3}{|c|}{ Best Hit to Arabidopsis (BLASTP) } \\
\hline & & & & & & & & & ID & Gene Name & E-Value \\
\hline BraA01g012490.3C & BrBGLU1 & 1290 & 430 & Chr 01 & $6,516,500$ & $6,519,450$ & 2950 & 11 & AT4G21760 & BGLU47 & 0 \\
\hline BraA01g029610.3C & BrBGLU2 & 1452 & 484 & Chr 01 & $19,673,693$ & $19,677,620$ & 3927 & 12 & AT1G61820 & BGLU46 & 0 \\
\hline BraA01g029670.3C & BrBGLU3 & 1551 & 517 & Chr 01 & $19,772,218$ & $19,775,132$ & 2914 & 12 & AT1G61810 & BGLU45 & 0 \\
\hline BraA01g032340.3C & BrBGLU4 & 873 & 291 & Chr 01 & $22,083,906$ & $22,086,749$ & 2843 & 8 & AT1G52400 & BGLU18 & $6.38 \times 10^{-85}$ \\
\hline BraA01g034680.3C & BrBGLU5 & 1545 & 515 & Chr 01 & $23,747,770$ & $23,750,431$ & 2661 & 12 & AT3G18080 & BGLU44 & 0 \\
\hline BraA01g034690.3C & BrBGLU6 & 1464 & 488 & Chr 01 & $23,754,677$ & $23,757,145$ & 2468 & 10 & AT3G18070 & BGLU43 & 0 \\
\hline BraA01g040820.3C & BrBGLU7 & 1374 & 458 & Chr 01 & $27,455,063$ & $27,458,182$ & 3119 & 12 & AT3G09260 & BGLU23 & 0 \\
\hline BraA01g041990.3C & BrBGLU8 & 1926 & 642 & Chr 01 & $28,048,456$ & $28,052,048$ & 3592 & 9 & AT3G06510 & BGLU48 & 0 \\
\hline BraA01g043570.3C & BrBGLU9 & 1566 & 522 & Chr 01 & $28,909,991$ & $28,913,218$ & 3227 & 13 & AT3G03640 & BGLU25 & 0 \\
\hline BraA02g023150.3C & BrBGLU10 & 1653 & 551 & Chr 02 & $13,570,012$ & $13,572,993$ & 2981 & 13 & AT1G75940 & BGLU20 & 0 \\
\hline BraA03g011770.3C & BrBGLU11 & 894 & 298 & Chr 03 & $5,059,601$ & $5,062,343$ & 2742 & 8 & AT1G45191 & BGLU1 & $2.53 \times 10^{-60}$ \\
\hline BraA03g011780.3C & BrBGLU12 & 1023 & 341 & Chr 03 & $5,063,808$ & $5,066,446$ & 2638 & 12 & AT1G60090 & BGLU4 & $9.15 \times 10^{-82}$ \\
\hline BraA03g024570.3C & BrBGLU13 & 846 & 282 & Chr 03 & $12,073,161$ & $12,075,780$ & 2619 & 10 & AT4G22100 & BGLU3 & $5.01 \times 10^{-68}$ \\
\hline BraA03g033950.3C & BrBGLU14 & 1398 & 466 & Chr 03 & $16,798,347$ & $16,801,182$ & 2835 & 11 & AT3G09260 & BGLU23 & 0 \\
\hline BraA03g041420.3C & BrBGLU15 & 729 & 243 & Chr 03 & $20,778,980$ & $20,781,062$ & 2082 & 5 & AT4G22100 & BGLU3 & $2.54 \times 10^{-61}$ \\
\hline BraA03g041430.3C & BrBGLU16 & 669 & 223 & Chr 03 & $20,781,085$ & $20,782,278$ & 1193 & 7 & AT1G60090 & BGLU4 & $3.4 \times 10^{-100}$ \\
\hline BraA03g049730.3C & BrBGLU17 & 1563 & 521 & Chr 03 & $25,428,252$ & $25,430,947$ & 2695 & 12 & AT4G21760 & BGLU47 & 0 \\
\hline BraA04g000610.3C & BrBGLU18 & 1431 & 477 & Chr 04 & 408,734 & 411,303 & 2569 & 11 & AT4G27830 & BGLU10 & 0 \\
\hline BraA04g002030.3C & BrBGLU19 & 1497 & 499 & Chr 04 & $1,226,615$ & $1,230,317$ & 3702 & 12 & AT3G60140 & BGLU30 & 0 \\
\hline BraA04g002040.3C & BrBGLU20 & 2058 & 686 & Chr 04 & $1,238,401$ & $1,245,729$ & 7328 & 18 & AT3G60120 & BGLU27 & $2.2 \times 10^{-149}$ \\
\hline BraA04g010020.3C & BrBGLU21 & 1341 & 447 & Chr 04 & $7,880,007$ & $7,883,680$ & 3673 & 13 & AT5G36890 & BGLU42 & 0 \\
\hline BraA04g020960.3C & BrBGLU22 & 891 & 297 & Chr 04 & $15,776,965$ & $15,780,643$ & 3678 & 8 & AT5G44640 & BGLU13 & $1.5 \times 10^{-105}$ \\
\hline BraA04g023640.3C & BrBGLU23 & 1638 & 546 & Chr 04 & $17,341,351$ & $17,344,539$ & 3188 & 9 & AT2G32860 & BGLU33 & 0 \\
\hline BraA04g031090.3C & BrBGLU24 & 1233 & 411 & Chr 04 & $21,218,491$ & $21,221,708$ & 3217 & 12 & AT3G60120 & BGLU27 & 0 \\
\hline BraA04g031100.3C & BrBGLU25 & 1380 & 460 & Chr 04 & $21,229,282$ & $21,232,323$ & 3041 & 11 & AT5G24550 & BGLU32 & 0 \\
\hline BraA04g031130.3C & BrBGLU26 & 267 & 89 & Chr 04 & $21,248,071$ & $21,248,622$ & 551 & 4 & AT2G44450 & BGLU15 & $5.66 \times 10^{-85}$ \\
\hline BraA04g031140.3C & BrBGLU27 & 525 & 175 & Chr 04 & $21,249,497$ & $21,251,177$ & 1680 & 6 & AT2G44450 & BGLU15 & $1.4 \times 10^{-110}$ \\
\hline BraA05g004330.3C & BrBGLU28 & 1623 & 541 & Chr 05 & $2,194,953$ & $2,197,671$ & 2718 & 11 & AT3G60120 & BGLU27 & 0 \\
\hline BraA05g004340.3C & BrBGLU29 & 1527 & 509 & Chr 05 & $2,201,548$ & $2,211,165$ & 9617 & 12 & AT2G44450 & BGLU15 & 0 \\
\hline BraA05g004350.3C & BrBGLU30 & 1518 & 506 & Chr 05 & $2,216,705$ & $2,220,674$ & 3969 & 12 & AT5G44640 & BGLU13 & 0 \\
\hline BraA05g004360.3C & BrBGLU31 & 1326 & 442 & Chr 05 & $2,223,901$ & $2,227,525$ & 3624 & 9 & AT2G44460 & BGLU28 & $2.7 \times 10^{-131}$ \\
\hline BraA05g004370.3C & BrBGLU32 & 1155 & 385 & Chr 05 & $2,245,448$ & $2,248,087$ & 2639 & 7 & AT3G60140 & BGLU30 & $2.1 \times 10^{-140}$ \\
\hline BraA05g004380.3C & BrBGLU33 & 1281 & 427 & Chr 05 & $2,255,905$ & $2,258,985$ & 3080 & 11 & AT5G24540 & BGLU31 & $5.1 \times 10^{-145}$ \\
\hline BraA05g004390.3C & BrBGLU34 & 1545 & 515 & Chr 05 & $2,261,685$ & $2,270,997$ & 9312 & 11 & AT2G44490 & BGLU26 & 0 \\
\hline
\end{tabular}


Table 1. Cont.

\begin{tabular}{|c|c|c|c|c|c|c|c|c|c|c|c|}
\hline \multirow{2}{*}{ Locus ID } & \multirow{2}{*}{ Gene Name } & \multirow{2}{*}{$\begin{array}{l}\text { CDS Length } \\
\text { (bp) }\end{array}$} & \multirow{2}{*}{$\begin{array}{c}\text { Protein } \\
\text { Length (aa) }\end{array}$} & \multirow{2}{*}{ Chromosome } & \multirow{2}{*}{ Gene Start } & \multirow{2}{*}{ Gene End } & \multirow{2}{*}{$\begin{array}{c}\text { gDNA } \\
\text { Length (bp) }\end{array}$} & \multirow{2}{*}{$\begin{array}{l}\text { No. of } \\
\text { Exons }\end{array}$} & \multicolumn{3}{|c|}{ Best Hit to Arabidopsis (BLASTP) } \\
\hline & & & & & & & & & ID & Gene Name & E-Value \\
\hline BraA05g012860.3C & BrBGLU35 & 1536 & 512 & Chr 05 & $7,011,962$ & $7,015,388$ & 3426 & 11 & AT2G32860 & BGLU33 & $2.3 \times 10^{-162}$ \\
\hline BraA05g012870.3C & BrBGLU36 & 957 & 319 & Chr 05 & $7,023,185$ & $7,028,485$ & 5300 & 8 & AT2G32860 & BGLU33 & $4.3 \times 10^{-102}$ \\
\hline BraA05g015060.3C & BrBGLU37 & 1461 & 487 & Chr 05 & $8,601,511$ & $8,604,284$ & 2773 & 13 & AT5G36890 & BGLU42 & 0 \\
\hline BraA05g017770.3C & BrBGLU38 & 1278 & 426 & Chr 05 & $10,758,114$ & $10,760,718$ & 2604 & 11 & AT1G52400 & BGLU18 & 0 \\
\hline BraA05g033960.3C & BrBGLU39 & 1434 & 478 & Chr 05 & $24,329,347$ & $24,333,054$ & 3707 & 12 & AT4G27830 & BGLU10 & 0 \\
\hline BraA05g037140.3C & BrBGLU40 & 1332 & 444 & Chr 05 & $25,685,745$ & $25,688,976$ & 3231 & 11 & AT3G09260 & BGLU23 & 0 \\
\hline BraA05g037150.3C & BrBGLU41 & 1374 & 458 & Chr 05 & $25,691,345$ & $25,694,889$ & 3544 & 12 & AT3G09260 & BGLU23 & 0 \\
\hline BraA05g038920.3C & BrBGLU42 & 1782 & 594 & Chr 05 & $26,547,600$ & $26,550,524$ & 2924 & 11 & AT3G06510 & BGLU48 & 0 \\
\hline BraA06g002000.3C & BrBGLU43 & 1347 & 449 & Chr 06 & $1,220,707$ & $1,223,925$ & 3218 & 12 & AT1G52400 & BGLU18 & $1.5 \times 10^{-173}$ \\
\hline BraA06g011040.3C & BrBGLU44 & 1080 & 360 & Chr 06 & $5,995,931$ & $6,000,341$ & 4410 & 8 & AT3G21370 & BGLU19 & 0 \\
\hline BraA06g 024630.3C & BrBGLU45 & 1599 & 533 & Chr 06 & $17,098,530$ & $17,100,946$ & 2416 & 4 & AT5G44640 & BGLU13 & 0 \\
\hline BraA06g038720.3C & BrBGLU46 & 312 & 104 & Chr 06 & $25,758,774$ & $25,759,164$ & 390 & 2 & AT4G22100 & BGLU3 & $8 \times 10^{-52}$ \\
\hline BraA07g008030.3C & BrBGLU47 & 1434 & 478 & Chr 07 & $8,145,282$ & $8,147,911$ & 2629 & 11 & AT1G60090 & BGLU4 & 0 \\
\hline BraA07g008050.3C & BrBGLU48 & 1428 & 476 & Chr 07 & $8,161,734$ & $8,164,666$ & 2932 & 12 & AT4G22100 & BGLU3 & 0 \\
\hline BraA07g011940.3C & BrBGLU49 & 765 & 255 & Chr 07 & $11,620,825$ & $11,623,618$ & 2793 & 8 & AT3G62750 & BGLU8 & $3.75 \times 10^{-29}$ \\
\hline BraA07g024150.3C & BrBGLU50 & 1545 & 515 & Chr 07 & $18,998,283$ & $19,001,907$ & 3624 & 12 & АT3G60130 & BGLU16 & 0 \\
\hline BraA08g002600.3C & BrBGLU51 & 1515 & 505 & Chr 08 & $1,915,015$ & $1,917,839$ & 2824 & 13 & AT1G47600 & BGLU34 & 0 \\
\hline BraA08g $008860.3 \mathrm{C}$ & BrBGLU52 & 408 & 136 & Chr 08 & $7,848,512$ & $7,850,189$ & 1677 & 4 & AT3G09260 & BGLU23 & $1.03 \times 10^{-75}$ \\
\hline BraA08g014870.3C & BrBGLU53 & 930 & 310 & Chr 08 & $12,301,355$ & $12,303,970$ & 2615 & 7 & AT4G22100 & BGLU3 & $3.9 \times 10^{-127}$ \\
\hline BraA08g $025770.3 \mathrm{C}$ & BrBGLU54 & 1506 & 502 & Chr 08 & $18,552,796$ & $18,556,470$ & 3674 & 11 & AT1G26560 & BGLU40 & 0 \\
\hline BraA09g018020.3C & BrBGLU55 & 1524 & 508 & Chr 09 & $11,385,273$ & $11,386,948$ & 1675 & 2 & AT5G44640 & BGLU13 & 0 \\
\hline BraA09g038410.3C & BrBGLU56 & 1527 & 509 & Chr 09 & $30,292,880$ & $30,295,941$ & 3061 & 11 & AT1G26560 & BGLU40 & 0 \\
\hline BraA09g049950.3C & BrBGLU57 & 1542 & 514 & Chr 09 & $37,157,612$ & $37,160,275$ & 2663 & 10 & AT3G60120 & BGLU27 & 0 \\
\hline BraA09g049960.3C & BrBGLU58 & 1248 & 416 & Chr 09 & $37,164,182$ & $37,167,906$ & 3724 & 10 & AT3G60130 & BGLU16 & $2.2 \times 10^{-165}$ \\
\hline BraA09g049970.3C & BrBGLU59 & 1047 & 349 & Chr 09 & $37,169,340$ & $37,173,150$ & 3810 & 8 & АТЗG60130 & BGLU16 & $3.4 \times 10^{-165}$ \\
\hline BraA09g049980.3C & BrBGLU60 & 1326 & 442 & Chr 09 & $37,178,403$ & $37,186,233$ & 7830 & 11 & AT3G60140 & BGLU30 & $2.6 \times 10^{-179}$ \\
\hline BraA09g052040.3C & BrBGLU61 & 1461 & 487 & Chr 09 & $38,112,498$ & $38,115,245$ & 2747 & 11 & AT4G27830 & BGLU10 & 0 \\
\hline BraA09g052050.3C & BrBGLU62 & 1176 & 392 & Chr 09 & $38,116,332$ & $38,118,944$ & 2612 & 11 & AT4G27830 & BGLU10 & $9.8 \times 10^{-140}$ \\
\hline BraA10g001490.3C & BrBGLU63 & 1281 & 427 & Chr 10 & 776,354 & 778,932 & 2578 & 11 & AT1G02850 & BGLU11 & 0 \\
\hline BraA10g012660.3C & BrBGLU64 & 1569 & 523 & Chr 10 & $10,414,966$ & $10,417,449$ & 2483 & 11 & AT5G54570 & BGLU41 & 0 \\
\hline
\end{tabular}




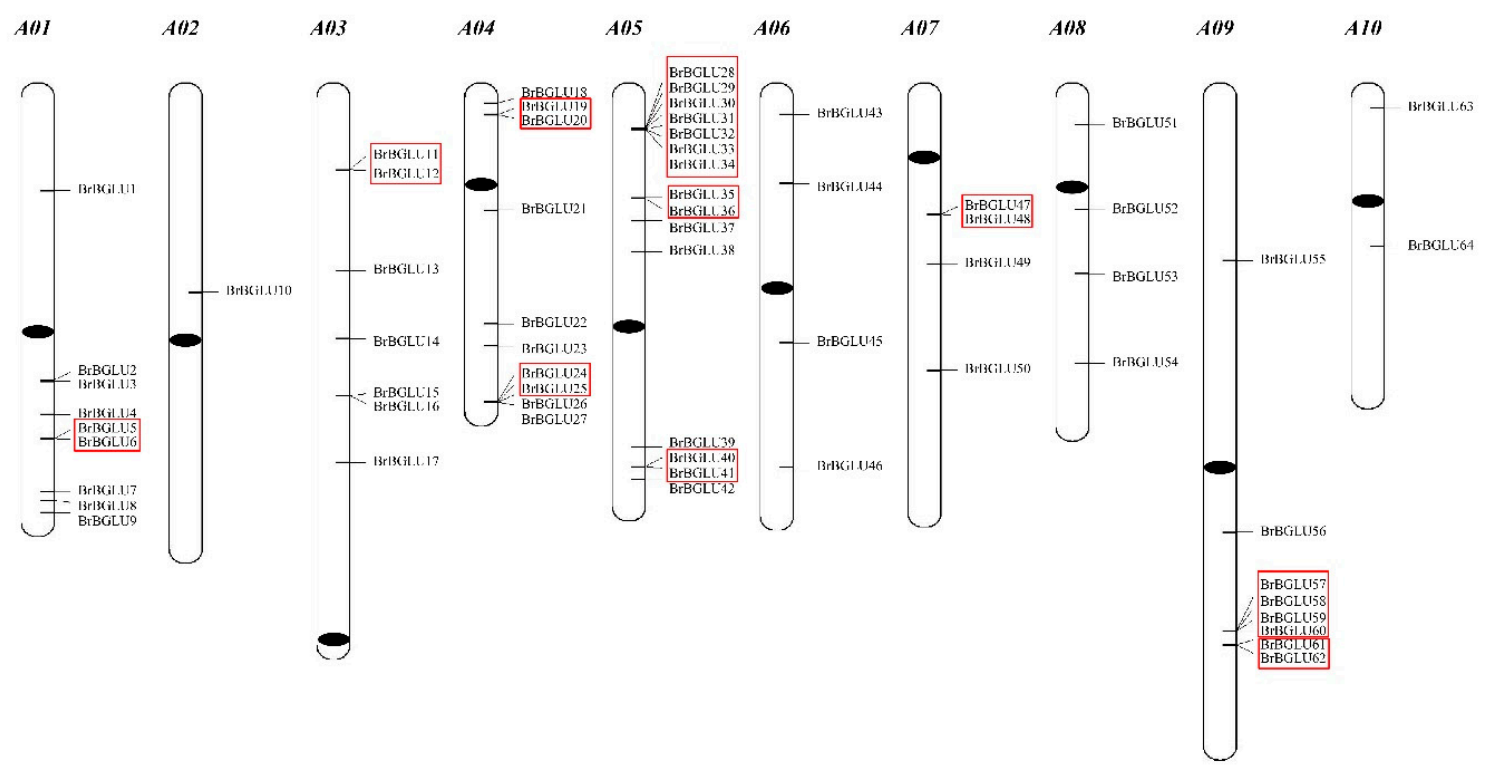

Figure 1. Chromosomal distribution of the $64 \mathrm{BrBGLU}$ genes identified in this study. The chromosome number is indicated above each chromosome. Ten clusters of BrBGLUs are indicated in red boxes. Black ovals on each chromosome represent the centromeric regions.

\subsection{Phylogenetic and Gene Structure Analysis of BrBGLUs}

To understand the evolutionary relationship of the BrBGLU genes, phylogenetic analysis of the BrBGLU and AtBGULU genes was conducted. To obtain AtBGLUs, HMM searching was performed by using all of the putative protein sequences of the Arabidopsis genome (ARAPORT11, https://www. araport.org) as queries. A total of $48 A t B G L U$ genes were obtained, which agrees with the results of a previous study [6]. The 64 BrBGLUs and 48 AtBGLUs protein sequences were aligned using ClustaX2 [31]. An unrooted phylogenetic tree was constructed for the 64 BrBGLUs and 48 AtBGLUs, using the NJ method in MEGA6 with a Poisson model. All BGLU proteins were classified into 10 distinct subgroups, namely, BGLU-a to BGLU-j (Figure 2). The results of the phylogenetic analysis were relatively similar to the findings of a previous study using Arabidopsis [6], with a few exceptions. All B. rapa and Arabidopsis proteins are grouped into 10 subgroups, whereas Arabidopsis subgroups 8 and 9 were combined into a subgroup GH1-c in our analysis. In addition, AtBGLU48 (SENSITIVE TO FREEZING 2, SFR2), which belongs to a distinct lineage from 10 subgroups in a previous study [6,32], was incorporated into the GH1-j subgroup, together with BrBGLU8 and BrBGLU42 (Figure 2).

Phylogenetic analysis generated an interesting finding, that the clustering or groupings of genes were related to the chromosomal locus or function. Based on the functions of the AtBGLUs, flavonol accumulation (AtBGLU1-6) and anthocyanin glucosyltransferase (AtBGLU7-11)-related genes were highlighted by subgroup GH1-a [10-12]; flavonoid utilization-related AtBGLUs (AtBGLU12-17) were represented by the GH1-e subgroup [10,13]; myrosinase-encoding AtBGLUs (AtBGLU34-39) belonged to the GH1-d subgroup [14-16], and scopolin hydrolysis-related AtBGLUs (AtBGLU21-23) were grouped into GH1-i [21,22]. Most of the genes within the same clusters on a chromosome were grouped into the same subfamily, which is similar to the findings using Arabidopsis, i.e., BrBGLU5/6, BrBGLU11/12, BrBGLU31/32/33, BrBGLU40/41, BrBGLU58/59, and BrBGLU61/62. This clustering indicates that the $B G L U$ genes may have evolved from an ancestral gene via gene duplication. However, BrBGLU51 was grouped with six AtBGLUs (AtBGLU34/35/36/37/38/39) in the GH1-d subgroup, indicating the possible loss of some $B G L U$ genes in B. rapa. 


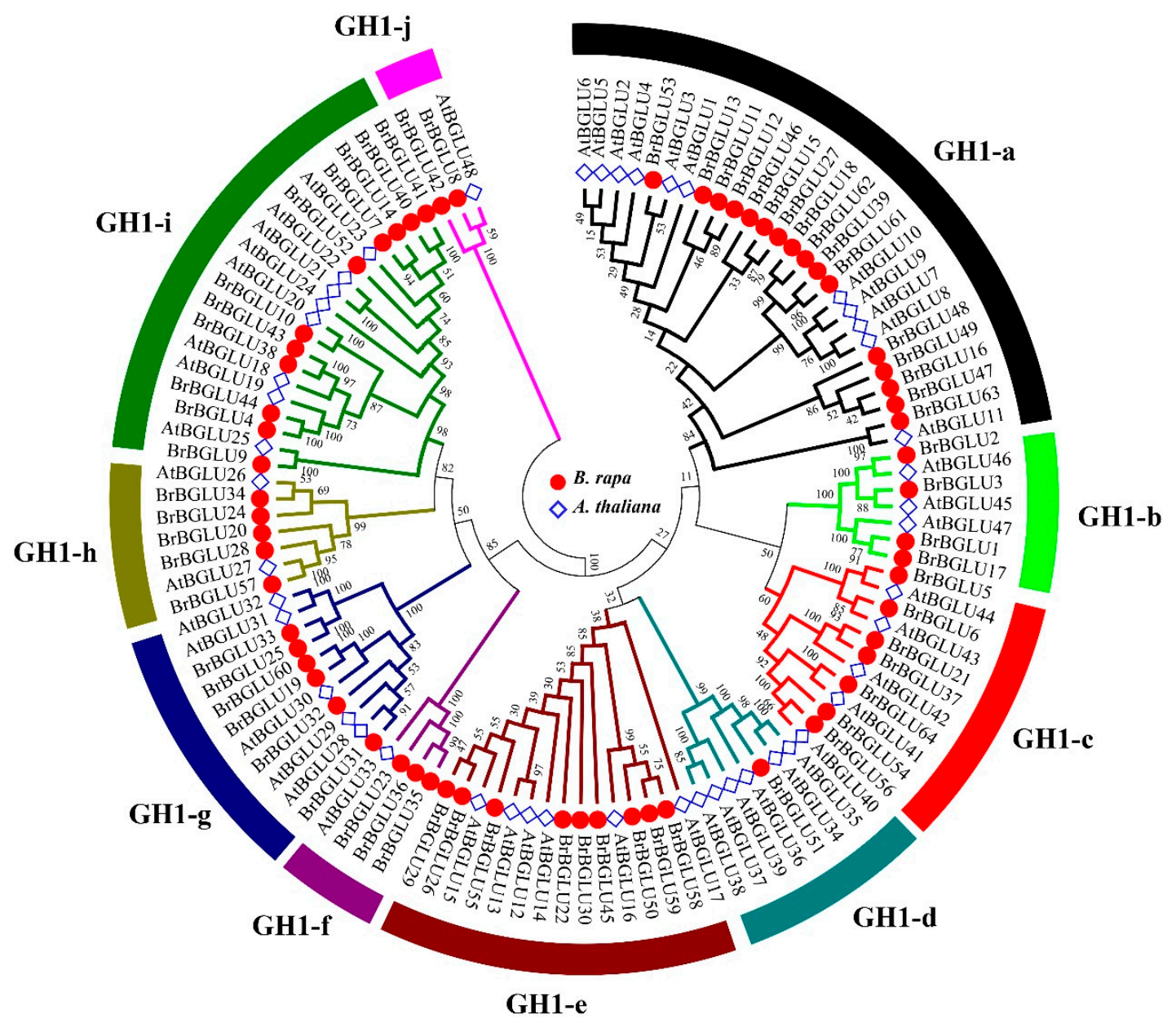

Figure 2. Phylogenetic reconstruction of GH1 genes of Arabidopsis and Brassica rapa. Multiple sequence alignment of GH1 proteins was performed using ClustalX2 with default parameters. The unrooted phylogenetic tree was constructed by MEGA 6 with the neighbor-joining (NJ) methods using the following parameters: bootstrap values (1,000 replicates) and Poisson model. The tree is divided into 11 phylogenetic subgroups, designated as GH1-a to GH1-k. Members of Arabidopsis and B. rapa are denoted by blue squares and red circles.

Gene structure was commonly diversified during the evolution of the large number of gene families. To expand our knowledge of BrBGLUs in relation to evolution and functional diversification, the gene structures of the BrBGLUs were analyzed on the basis of exon-intron organization, using GSDS 2.0 [33]. The BrBGLUs exhibited 12 distinct exon-intron organization patterns, and the most common organization was 11 exons separated by 10 introns, presenting 19 members (Table 1 and Figure 3). Most genes contained more than two introns, except for BrBGLU46 and BrBGLU55, indicating the possible occurrence of alternative splicing during gene expression. The AtBGLUs exhibited 10 distinct exon-intron organization patterns, and the pattern with 13 exons was the most common [6]. This analysis is consistent with Arabidopsis and rice, where the intron size and number of the BGLUs genes are highly variable $[5,6]$. 


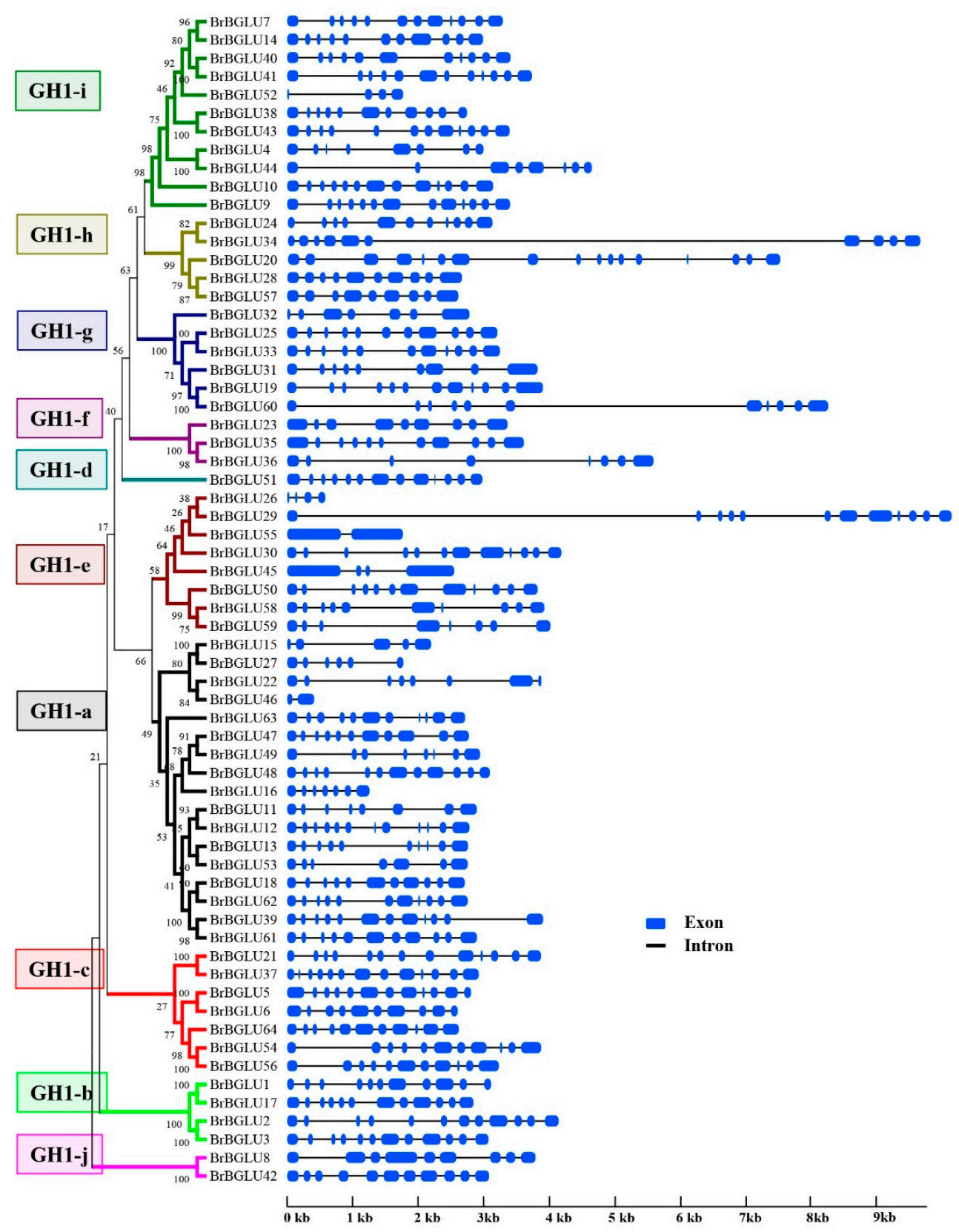

Figure 3. Exon-intron organization of BrBGLUs in different subgroups. Exons and introns are represented by blue boxes and black lines, respectively. The phylogenetic tree of each subfamily was constructed using MEGA6, as described in Figure 1.

\subsection{Identification of BrBGLU Genes Involved in Pollen Development}

Rice TDR (Tapetum Degeneration Retardation) mutant alters BGLU1 expression with flower specificity [28], and BGLU1 and BGLU13 are found to be related to male organ development in Calamus palustris [34]. These previous reports lead to a hypothesis that BrBGLUs are involved in pollen development. To test this hypothesis, the previously published microarray data relating to male sterility in B. rapa [29] were re-annotated, using the improved B. rapa genome (version 3.0) [35] and analyzed based on pollen development (Table S1). A total of 36 BrBGLUs, represented by 88 probes (or 88 ESTs) showed significant hybridization values, of which 12 BrBGLUs showed over two-fold change in expression levels between fertile and sterile floral buds: six members were upregulated, and members were downregulated in fertile buds. Among these genes, four upregulated genes (BrBGLU10/AtBGLU20, BrBGLU15/AtBGLU3, BrBGLU16/AtBGLU4, and BrBGLU64/AtBGLU41) and 
two downregulated genes (BrBGLU2/AtBGLU46 and BrBGLU19/AtBGLU30) were described as good candidates that were associated with pollen development. The function of all four upregulated genes has not been known up to now, but at least three, BrBGLU10, BrBGLU15, and BrBGLU64 appeared to be related to pollen wall development. In particular, we further analyzed BrBGLU10/AtBGLU20, as these showed hundred-fold changes between fertile and sterile buds.

\subsection{Analysis of the Putative Functions of BrBGLU10/AtBGLU20 in Pollen Development}

To gain more insights into the functions of the BrBGLUs during pollen development, BrBGLU10, which was highly and specifically expressed in fertile buds, was selected for further analysis. AtBGLU20, the Arabidopsis ortholog of BrBGLU10, was initially named as ATA27, which is one of the A. thaliana anther-specific expressed genes [36]. To confirm the expression patterns of BrBGLU10 and AtBGLU20, RT-PCR was conducted (Figure 4A,B). The expression level of BrBGLU10 was specifically detected at the F1-F3 stages, with highest levels at the F2 stage, representing the tetrad stage, and AtBGLU20 was specifically expressed before floral stage 12 . The RT-PCR results might imply its important role in pollen development.

A

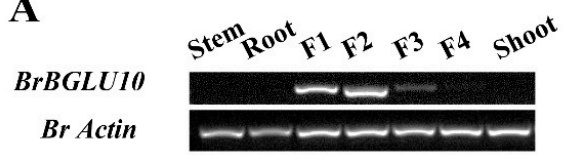

C

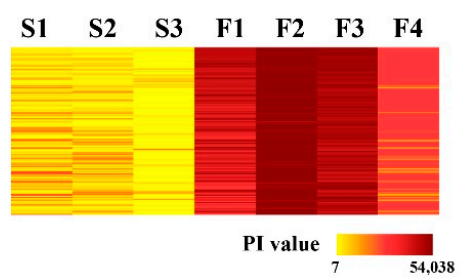

$\mathbf{E}$

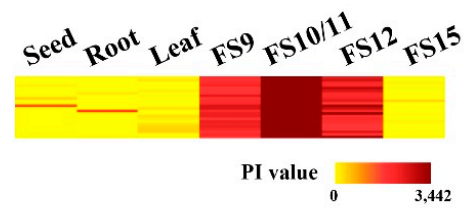

B

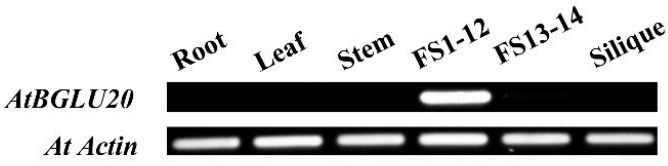

D

GO_ACC. Description $P$-value

GO:0010208 Pollen wall assembly 2.20E-14 GO:0010584 Pollen exine formation 2.20E-14 GO:0009555 Pollen development 2.10E-07 GO:0006629 Lipid metabolic process 1.70E-05 GO:0048229 Gametophyte development 1.70E-04

\section{F}

GO_ACC. Description P-value

GO:0010584 Pollen exine formation $\quad 3.20 \mathrm{E}-24$

GO:0010208 Pollen wall assembly $\quad 3.20 \mathrm{E}-24$

GO:0009555 Pollen development 2.60E-16

GO:0048229 Gametophyte development $6.60 \mathrm{E}-14$

Figure 4. Analysis of expression of BrBGLU10 and AtBGLU20, and Gene Ontology (GO) enrichment of co-expressed genes. A, Expression of BrBGLU10 in different tissues and floral bud stages in B. rapa. B, Expression of AtBGLU20 in different tissues and floral bud stages in Arabidopsis. C, Expression patterns of BrBGLU10 and its co-expressed genes in sterile and fertile B. rapa floral buds, based on previously published microarray data [29]. D, GO enrichment analysis of genes co-expressed with BrBGLU10. E, Expression pattern of AtBGLU20 and its co-expressed genes in various tissues of Arabidopsis, which was performed using the Arabidopsis eFP Browser (http:/ /bar.utoronto.ca/efp/cgibin/efpWeb.cgi). F, GO enrichment analysis of genes co-expressed with AtBGLU20. S1-S3 represent the floral buds from male-sterile B. rapa. S1, before the tetrad stage. S2, after the tetrad stage. S3, containing aberrant pollen grains. F1-F4 indicate fertile B. rapa floral buds before the tetrad stage (F1), at the tetrad stage (F2), after the tetrad stage, but before containing mature pollen (F3), and containing mature pollen (F4). For Arabidopsis, FS1-12, flower stage 1 to stage 12; FS13-14, flower stage 13 to stage 14. PI, probe intensity.

To demonstrate similar or conserved functions between BrBLU10 and AtBGLU20, we isolated the co-expressed genes of BrBGLU10, using microarray data [29] and AtBGLU20 from the Arabidopsis eFP Browser (http:/ / bar.utoronto.ca/efp/cgi-bin/efpWeb.cgi) [37]. With the Pearson's correlation coefficient (PCC) value above $0.90,183$ probes (107 genes) and 25 genes were determined to be 
co-expressed with BrBGLU10 and AtBGLU20, respectively (Figure 4C, E; Tables S2-S3). BrBGLU10 and its co-expressers were upregulated at the fertile floral bud stage, and the highest expression level was detected at the F2 stage (Figure 4C), suggesting that BrBGLU10 plays a role during pollen development, especially from the tetrad stage to that before the mature pollen stage. In Arabidopsis, flower and stamen development processes were divided into 14 stages and 12 stages, respectively [37-39]. AtBGLU20 and its co-expressers were represented by a high probe intensity (PI) value at flower stages (FS) 9 to 12, indicating that AtBGLU20 plays a role in Arabidopsis pollen development (Figure 4E). We also conducted Gene Ontology (GO) enrichment analysis to provide more information on the function of BrBGLU10 and AtBGLU20 (Figure 4D,F). The results showed that genes involved in pollen exine formation and pollen wall assembly were highly over-represented among genes co-expressed with BrBGLU10 and AtBGLU20. Taken together, our analysis indicated that BrBGLU10 and AtBGLU20 may be required for pollen development in both B. rapa and Arabidopsis.

To validate $A t B G L U 20$ function in the pollen development, we generated knockdown mutants of AtBGLU20 by introducing antisense constructs under the control of the CaMV35S promoter (Figure 5A). After screening, four independent knockdown lines were obtained with expression levels ranging from $55 \%$ to $85 \%$ (Figure $5 \mathrm{~B}$ ). However, the AtBGLU20 downregulated plants showed normal vegetative growth based on morphology (Figure $5 \mathrm{C}$ ), but produced defective pollen grains relative to the wild-type plants (Figure 5D). These results indicated that normal pollen development in Arabidopsis requires sufficient amounts of AtBGLU20. All data obtained from gene expression, co-expression analysis, and transgenesis led to the conclusion that AtBGLU20 and BrBGLU10 may have indispensable functions in pollen development in both Arabidopsis and B. rapa, respectively.

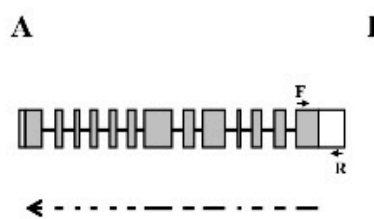

B

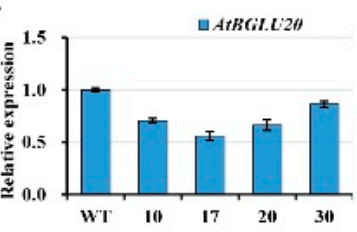

C

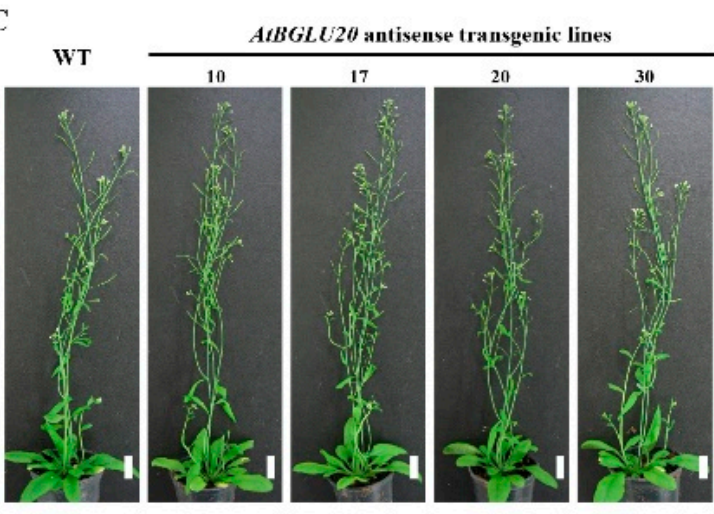

D

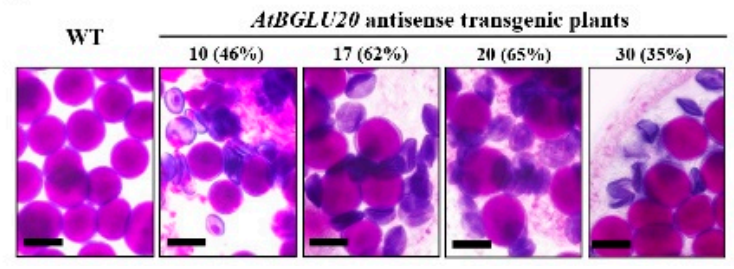

Figure 5. Analysis of WT and AtBGLU20 antisense knockdown mutant Arabidopsis plants. A. Schematic representation of the $A t B G L U 20$ gene structure and DNA fragment regions for antisense constructs. The white box indicates the UTR region; gray boxes are exons; lines represent introns. The single arrow indicates the antisense orientation of the fragments in the constructs. F and R indicate the primer 
positions used in qRT-PCR analysis. B, Analysis of the expression levels of AtBGLU20. Expression was normalized to that of AtACT7, and represented relative to the expression levels of the WT. Error bars represent the SD of three biological replicates. C, Morphologies of wild-type Arabidopsis plants and AtBGLU20 knockdown transgenic plants, which showed no obvious differences in vegetative growth. Bar $=20 \mathrm{~mm}$. D, Mature pollen grain of WT and AtBGLU20 transgenic plants stained with modified Alexander solution (Peterson et al., 2010). The well-developed pollen grains were stained red. Bar $=20 \mu \mathrm{m}$. WT, wild-type. 10,17, 20, and 30 indicate four independent transgenic lines. The number in the parentheses indicate the percentages of defective pollen grains.

\section{Discussion}

\subsection{Identification and Analysis of BrBGLUs}

GH1 family genes play an important role in regulating abiotic and biotic stress responses, as well as various developmental processes in plants $[9,12,14,18,23,40]$. Based on the results of an increase in the number of whole genome sequences from a large number of species, genome-wide analysis of various gene families has been extensively performed. However, genome-wide identification and characterization of the GH1 gene family has only been reported in a few plant species, and there is no information on Brassica species, which are important crops for production of functional foods, as well as health-promoting compounds. In this study, the isolation of BrBGLUs from $B$. rapa genome (Figure 1), the distribution of BrBGLU genes on chromosomes (Figure 1), phylogenetic analysis (Figure 2), and exon-intron structures (Figure 3) provides substantial information on the functions and roles of these genes.

Compared with the 49 AtBGLUs and 37 OsBGLUs in Arabidopsis and Rice, respectively [9], $64 \mathrm{BrBGLU}$ s were isolated from the $B$. rapa genome, which is the largest number so far that has been reported in plants (Figure 1). The high number of BGLU family members in B. rapa could be related to the genome triplication event in this lineage [41]. To adapt different new functions that are suitable for changes in the environment, gene structure was commonly diversified during the evolution of multigene families [42]. For BGLUs, 13 exon-12 intron organization was considered as the ancestral gene structure, with the loss of certain introns leading to other gene structures [6]. The exons present in BrBGLUs varied from 2 to 13, and the most common organization was 11 exons (Figure 3). The introns in Arabidopsis vary from 0 to 13 [6]. This results suggested that little diversity exists in the gene structure of BrBGLUs when compared to AtBGLUs.

BrBGLUs may have originated from Arabidopsis, although duplication, gene loss, and functional diversification may have also occurred. This is supported by the fact that BGLUs from both species could be grouped into 10 subfamilies, with tandem arrays, as defined by Singh et al., 2013 [39], although some families were re-grouped or diverged into other subgroups. Figure 2 shows that AtBGLU subfamilies 8 and 9 [6] were incorporated into one B. rapa subfamily, GH1-c, and BrBGLU51 is composed of GH1-d with six AtBGLUs (AtBGLU34/35/36/37/38/39), indicating the loss of some BGLUs in $B$. rapa. This phenomenon may result from the rapid evolution of genes similar to that previously observed between Arabidopsis and rice [5]. One more interesting finding was that AtBGLU48 (SFR2) was incorporated into the GH1-j subgroup, with BrBGLU8 and BrBGLU42 (Figure 2). AtBGLU42 is a $\beta$-glucosidase, but it is divergent from all other AtBGLUs, and more similar to several $\beta$-glycosidases from thermophilic archea and bacteria [32]. SFR2 is involved in the lipid remodeling of the outer chloroplast membrane during freezing tolerance $[43,44]$. Because two BrBGLUs in the GH1-j subgroup had identities between $85 \%$ and $87 \%$ with AtBGLU2, Brassica genes may have a similar function of freezing tolerance as that in AtSFR2, although this requires further investigation.

On the basis of Arabidopsis study, most subfamilies of BGLUs in Figure 2 may be associated with specific functions: GH1-a for flavonoid and anthocyanin metabolism, GH1-e for flavonoid utilization, GH1-d for myrosinases, and GH1-i for scopolin hydrolysis. At least 12 genes are known to be involved in flavonoid metabolism in GH1-a: AtBGLU1-6 for flavonol accumulation [10,11], AtBGLU7-11 as anthocyanin glucosyltransferases [10-12], and AtBGLU15 for flavonol bisglycoside 
catabolism under abiotic stress [13]. AtBGLU12-17 in the GH1-e subgroup code for flavonoid-utilizing BGLUs in legumes [10]. An examination of the functions of BrBGLUs that are clustered with AtBGLUs in subgroups GH1a and GH1-e may provide information and understanding into the regulation of flavonoid biosynthesis in Brassica species.

Several subfamilies may be related to abiotic and biotic stress resistance, such as GH1-b, GH1-c, GH1-d, GH1-f, and GH1-i. Myrosinases hydrolyze glucosinolates into active forms that are involved in plant defense against herbivory and pathogens, and in human health promotion [45-48]. AtBGLU26 and AtBGLU34-39 function as myrosinases [14-16]. Except for AtBGLU26 (GH1-h), most genes belong to the GH1-d subgroup (Figure 2). Understanding myrosinase function in Brassicaceae, which is rich in glucosinolates, may provide an excellent strategy for breeding health-promoting Brassica crops $[49,50]$. ABA also functions in stress responses, including drought stress. AtBGLU18 [17] and AtBGLU33 [18] hydrolyze glucose-conjugated $\mathrm{ABA}$, thereby increasing $\mathrm{ABA}$ levels and inducing $\mathrm{ABA}$ responses such as drought tolerance. However, these two proteins are separated into two subfamilies, implying the presence of more BGLUs for the regulation of ABA levels. Scopolin is one of the coumarins produced in roots [51], and it plays a role in the defense against pathogen attack and abiotic stresses $[19,20]$. Three $\beta$-glucosidases that hydrolyze scopolin and their encoding genes (AtBGLU21, 22 and 23) have been characterized [21,22]. The GH1-i subfamily includes these three genes and 11 BrBGLUs, which should be examined in relation to scopolin production. The GH1-b subfamily includes two monolignol glucoside hydrolases (AtBGLU45 and AtBGLU46) that control lignin content [23]. Because OsBGLU14, 16 , and 18 are involved in lignin biosynthesis with monolignol $\beta$-glucosidase activity and compensate for the Arabidopsis bglu45 mutant [52], BrBGLUs in this subfamily may play similar roles. AtBGLU42 in GH1-c is involved in the induction of systemic resistance to bacterial disease, and the release of iron-mobilizing phenolic metabolites under iron deficiency [24]. Several genes in this subfamily would thus be expected to contribute to eliciting defense responses. All of this information may contribute to future research directions in relation to BrBGLUs.

\subsection{The Potential Functions of BrBGLUs During Pollen Development}

Previous studies on rice and other plant species have indicated that $\beta$-glucosidases play roles in pollen development $[34,36,53]$. To identify the BrBGLUs responsible for pollen development, the previously published microarray data relating to male sterility in B. rapa were re-annotated and re-analyzed. Among the $36 \mathrm{BrBGLUs}, 12 \mathrm{BrBGLUs}$ showed over a two-fold change between fertile and sterile floral buds (Table S1). However, six genes (four upregulated and two downregulated genes) were more extensively studied in terms of their role in pollen development. We selected one BrBGLU10 for investigation, the homolog of AtBGLU20, which showed hundreds-fold changes in its expression.

We examined the expression levels of BrBGLU10/AtBGLU20 and analyzed the co-expressed genes in both B. rapa and Arabidopsis (Figure 4). An assessment of expression levels strongly suggests that BrBGLU10/AtBGLU20 are involved in pollen development. The cellular contents from the degeneration of the tapetum supports pollen wall formation and subsequent pollen release [39]. Mutations in polysaccharide metabolism-related genes lead to defective pollen wall formation [26]. Glycoside hydrolase has been reported to be involved in the cell wall polysaccharide degradation [27]. The expression patterns of BrBGLU10 and AtBGLU20 suggest that they might play a role from the tetrad stage to mature pollen grains (Figure $4 \mathrm{~A}, \mathrm{~B}$ ), which corresponding to the tapetum degradation stage $[29,39]$. Co-expression analysis is a valuable approach for classifying and visualizing transcriptomic data to identify genes with similar cellular functions and regulatory pathways [54-56], although this is not always the case [57,58]. In plants, co-expression analysis under various experimental conditions has been used for predicting gene function $[55,59]$. Figure $4 C, D$ shows that this gene possibly regulates pollen development. In particular, GO annotation of co-expressed genes reflects that pollen wall and exine formation are influenced by BrBGLU10/AtBGLU20, indicating that the hydrolysis of glucose moieties is necessary for proper pollen development. 
Because BrBGLU10 had a high sequence identity with AtBGLU20 (87\% at the nucleotide level and $84 \%$ at the amino acid sequence level), both genes may thus have similar functions. Therefore, knocking down AtBGLU20 may provide direct evidence for its function in pollen development. Figure 5 shows that the suppression of $A t B G L U 20$ expression had no effect on plant growth and development, although this aborted pollen production. This result implies that BrBGLU10/AtBGLU20 are critical to pollen grain development.

\section{Materials and Methods}

\subsection{Plant Materials and Growth Conditions}

Seeds of B. rapa subsp. pekinensis (Chiifu) were germinated in Petri dishes in the dark at $2{ }^{\circ} \mathrm{C}$ for two days, then the germinated seeds were transferred to a $4{ }^{\circ} \mathrm{C}$ growth chamber with $16 \mathrm{~h}$ of light for 25 days to induce vernalization. After vernalization, the seedlings were transplanted into $15 \mathrm{~cm} \times 15 \mathrm{~cm} \times 18 \mathrm{~cm}$ pots containing potting soil and grown in a $23{ }^{\circ} \mathrm{C}$ growth chamber with $16 \mathrm{~h}$ of light. The floral buds were collected from 10 plants with three biological replicates, as previously described [29], and stored at $-70{ }^{\circ} \mathrm{C}$ until use. Root and shoot tissues were collected from three-week-old seedlings without vernalization. Stem tissue was sampled from the plants one week after bolting.

A. thaliana (L.) Heynh var. Columbia (Col-0) plants were grown under $140 \mu \mathrm{mol} / \mathrm{m}^{2} / \mathrm{s}$ light intensity at $23 \pm 1{ }^{\circ} \mathrm{C}$ with a long day cycle with $16 \mathrm{~h}$ of light for plant transformation. Seeds were sown in $55 \mathrm{~mm} \times 55 \mathrm{~mm}$ pots in potting soil, stratified for three days at $4{ }^{\circ} \mathrm{C}$, and then transferred to the growth room. The plants were then kept under a transparent polythene lid for one week to increase humidity and support equal germination. The plate-cultured seeds were sterilized with $30 \%$ bleach and 0.1\% Triton X-100 (Sigma, St. Louis, MO, USA), stratified for three days at $4{ }^{\circ} \mathrm{C}$, and sown in Petri dishes with dimensions of $100 \mathrm{~mm} \times 100 \mathrm{~mm} \times 20 \mathrm{~mm}$. The dishes contained half-strength MS media (Duchefa Biochemie, Netherlands) supplemented with $0.8 \%$ phytoagar and $1 \%$ sucrose.

\subsection{Antisense Constructs and Plant Transformation}

The full-length coding sequence of AtBGLU20 was cloned from first-strand complementary DNA (cDNA), using the primers BGLU20F (Table S4). Then, the fragments were inserted into T\&A cloning vectors (RBC T\&A cloning kit, Real Biotech Corporation, Taiwan). After confirmation of the $A t B G L U 20$ sequence in the T\&A vector by sequencing, the fragment was cloned into pCambina 3300-35S binary vectors and used in plant transformation. Col-0 were used for transformation with Agrobacterium tumefaciens GV3101 carrying the above binary plasmid using the floral dip method [60]. The transformants were selected on plates containing $25 \mathrm{mg} / \mathrm{mL}$ glufosinate in MS medium (Sigma, St. Louis, MO, USA), and also confirmed by genomic DNA PCR analysis.

\subsection{Reverse Transcription PCR and $q R T-P C R$}

Total RNA $(1 \mu \mathrm{g})$ from each sample was used in reverse transcription. First-strand cDNA was synthesized with a PrimeScript ${ }^{\mathrm{TM}}$ RT reagent kit with a gDNA Eraser kit (TaKaRa, Japan). The concentration of the synthesized cDNA was determined, and the cDNA was diluted to $20 \mathrm{ng} / \mu \mathrm{L}$ for PCR analysis. Semi-RT-PCR was performed, which consisted of denaturation at $94{ }^{\circ} \mathrm{C} 5 \mathrm{~min}$; followed by 25 cycles of $94{ }^{\circ} \mathrm{C}$ for $30 \mathrm{~s}, 55^{\circ} \mathrm{C}$ for $30 \mathrm{~s}$, and $72{ }^{\circ} \mathrm{C}$ for $60 \mathrm{~s}$. The qRT-PCR conditions were pre-denaturation at $95^{\circ} \mathrm{C}$ for $30 \mathrm{~s}$; followed by 30 cycles of $95^{\circ} \mathrm{C}$ for $5 \mathrm{~s}, 60^{\circ} \mathrm{C}$ for $20 \mathrm{~s}$, and $72{ }^{\circ} \mathrm{C}$ for $15 \mathrm{~s}$. All primer sequences used in this study are listed in Table S4. The semi-RT-PCR products were separated on $1.5 \%$ agarose gels, and stained with ethidium bromide. The qRT-PCR results were analyzed using the $2^{-\Delta \Delta C}$ Tethod, with three biological replicates. 


\subsection{Pollen Viability}

For pollen viability and pollen developmental progression, flowers collected from Col-0 and AtBGLU20 antisense transgenic plants were fixed in Carnoy's fixative (6:3:1 alcohol:chloroform:acetic acid) for $2 \mathrm{~h}$. Then, the anthers were detected and stained with a solution of Malachite green, acid fuchsin, and Orange $\mathrm{G}$ for approximately $12 \mathrm{~h}$, as previously described [61].

\subsection{Identification of BrBGLUs and Phylogenetic Tree Construction}

The protein sequence of 48 BGLU members were downloaded from TAIR (http://www. arabidopsis.org/tools/bulk/sequences/index.jsp) [6]. All putative protein sequences of B. rapa (version 3.0) were downloaded from BRAD (http:/ / brassicadb.org/brad/index.php) [35] and used as queries to search against the Hidden Markov Model (HMM) profile (Version 3.1b2) with the Pfam HMM library (Pfam 32.0) [62]. A total of 64 protein sequences with PF00232 (E value below $1 \mathrm{E}^{-5}$ ) were obtained, and these sequences were considered as BrBGLUs candidates and used for further analysis. Multiple sequence alignment of full-length BGLU proteins and phylogenetic tree construction were conducted using ClustalX2 [31]. The phylogenetic tree was generated by the MEGA6 program, using the neighbor-joining method with the 'pairwise deletion' option and 'Poisson correction' model, with a bootstrap test of 1000 replications [63].

\subsection{Chromosomal Location, Nomenclature, and Gene Duplication of BrBGLUs}

The position of each BrBGLU on $B$. rapa chromosomes was identified from BRAD (http:/ / brassicadb.org/brad/index.php). For nomenclature, the ' $B r^{\prime}$ for B. rapa was added, followed by BGLU, and numbered according to its position from top to bottom on $B$. rapa chromosomes 1-10. MCScanX software was used to search potentially duplicated BrBGLUs [64]. All of the putative protein sequences of $B$. rapa (version 3.0) were compared with themselves by BLASTP, with a tabular format and an e-value of $<10^{-5}$. Then, tandem, segmental, and dispersed duplications were identified using MCScanX, using default criteria.

\subsection{Co-Expression and Gene Ontology Enrichment Analysis}

AtBGLU20 was used as bait gene for genome-wide co-expression analysis to identify genes of similar function from Expression Angler [65]. BrBGLU10 was represented by two EST probes Brapa_ESTC004210 and Brapa_ESTC007739, which were used as bait for co-expression analysis. A cutoff threshold of 0.90 for the Pearson correlation coefficient was used. The expression pattern analysis was performed using the Arabidopsis eFP browser (http://bar.utoronto.ca/efp/cgi-bin/efpWeb. cgi) [37]. Clustering analysis for categorization was performed with the TIGR Multi-Experiment Viewer (http://www.tm4.org/mev.html). GO enrichment analysis was performed using agriGO (http://bioinfo.cau.edu.cn/agriGO/index.php) [66].

\subsection{Microarray Analysis}

To analyze the gene expression patterns of BrBGLUs in B. rapa during pollen development, the previously published microarray data relating to male sterility analysis were downloaded from NCBI's Gene Expression Omnibus (GSE47665) [29]. The microarray data were re-annotated using BLASTX by comparing with the newly improved B. rapa reference genome sequence (version 3.0) [35].

\section{Conclusions}

In conclusion, $64 \mathrm{BrBGLUs}$ have been identified in B. rapa genome, which were classified into 10 subgroups with Arabidopsis counterparts, and the GH1-i subgroup included putative pollen development-related BrBGLU10. Base on its known function in Arabidopsis, BrBGLUs may participate in various defense responses against biotic and abiotic stresses, flavonoid metabolism, and pollen 
development. This study has provided valuable information for a better understanding of BGLUs, and for their biotechnological application to crops.

Supplementary Materials: Supplementary materials can be found at http://www.mdpi.com/1422-0067/20/7/ $1663 /$ s1.

Author Contributions: Designed the research scheme: X.D. and Y.H. Performed the experiments: X.D. and Y.J. Analyzed the data: X.D., Y.J., and Y.H. Wrote the manuscript: X.D. and Y.H. All authors read and approved the final manuscript.

Funding: This research was funded by the National Science Foundation of China, 31601771 and the Applied Basic Research Project of Yunnan, 2017FB056.

Acknowledgments: We thank LetPub (www.letpub.com) for its linguistic assistance during the preparation of this manuscript.

Conflicts of Interest: The authors declare no conflict of interest.

$\begin{array}{ll}\text { Abbreviations } \\ \text { GH1 } & \text { Glycoside hydrolase family } 1 \\ \text { BGLUs } & \beta \text {-glycosidase genes } \\ \text { BrBGLUs } & \text { Brassica rapa } \beta \text {-glycosidase genes } \\ \text { ABA } & \text { abscisic acid } \\ \text { OsTDR } & \text { Tapetum Degeneration Retardation } \\ \text { HMM } & \text { Hidden Markov Model } \\ \text { GO } & \text { Gene Ontology } \\ \text { CDS } & \text { coding sequence }\end{array}$

\section{References}

1. Chandrasekar, B.; Colby, T.; Emran Khan Emon, A.; Jiang, J.; Hong, T.N.; Villamor, J.G.; Harzen, A.; Overkleeft, H.S.; van der Hoorn, R.A. Broad-range glycosidase activity profiling. Mol. Cell. Proteomics 2014, 13, 2787-2800. [CrossRef]

2. Cantarel, B.L.; Coutinho, P.M.; Rancurel, C.; Bernard, T.; Lombard, V.; Henrissat, B. The carbohydrate-active enzymes database (cazy): An expert resource for glycogenomics. Nucleic Acids Res. 2009, 37, D233-D238. [CrossRef]

3. Lombard, V.; Golaconda Ramulu, H.; Drula, E.; Coutinho, P.M.; Henrissat, B. The carbohydrate-active enzymes database (cazy) in 2013. Nucleic Acids Res. 2014, 42, D490-D495. [CrossRef] [PubMed]

4. Henrissat, B. A classification of glycosyl hydrolases based on amino acid sequence similarities. Biochem. J. 1991, 280, 309-316. [CrossRef] [PubMed]

5. Opassiri, R.; Pomthong, B.; Onkoksoong, T.; Akiyama, T.; Esen, A.; Ketudat Cairns, J.R. Analysis of rice glycosyl hydrolase family 1 and expression of os4bglu12 beta-glucosidase. BMC Plant Biol. 2006, 6, 33. [CrossRef] [PubMed]

6. Xu, Z.; Escamilla-Trevino, L.; Zeng, L.; Lalgondar, M.; Bevan, D.; Winkel, B.; Mohamed, A.; Cheng, C.L.; Shih, M.C.; Poulton, J.; et al. Functional genomic analysis of arabidopsis thaliana glycoside hydrolase family 1. Plant Mol. Biol. 2004, 55, 343-367. [CrossRef]

7. Gomez-Anduro, G.; Ceniceros-Ojeda, E.A.; Casados-Vazquez, L.E.; Bencivenni, C.; Sierra-Beltran, A.; Murillo-Amador, B.; Tiessen, A. Genome-wide analysis of the beta-glucosidase gene family in maize (zea mays 1. Var b73). Plant Mol. Biol. 2011, 77, 159-183. [CrossRef] [PubMed]

8. Zhao, L.; Liu, T.; An, X.; Gu, R. Evolution and expression analysis of the $\beta$-glucosidase (glu) encoding gene subfamily in maize. Genes Genom. 2012, 34, 179-187. [CrossRef]

9. Cao, Y.Y.; Yang, J.F.; Liu, T.Y.; Su, Z.F.; Zhu, F.Y.; Chen, M.X.; Fan, T.; Ye, N.H.; Feng, Z.; Wang, L.J.; et al. A phylogenetically informed comparison of gh1 hydrolases between arabidopsis and rice response to stressors. Front Plant Sci. 2017, 8, 350. [CrossRef] [PubMed]

10. Roepke, J.; Bozzo, G.G. Arabidopsis thaliana $\beta$-glucosidase bglu15 attacks flavonol 3-O- $\beta$-glucoside-7-O- $\alpha$-rhamnosides. Phytochemistry 2015, 109, 14-24. [CrossRef] [PubMed] 
11. Ishihara, H.; Tohge, T.; Viehöver, P.; Fernie, A.R.; Weisshaar, B.; Stracke, R. Natural variation in flavonol accumulation in arabidopsis is determined by the flavonol glucosyltransferase bglu6. J. Exp. Bot. 2016, 67, 1505-1517. [CrossRef] [PubMed]

12. Miyahara, T.; Sakiyama, R.; Ozeki, Y.; Sasaki, N. Acyl-glucose-dependent glucosyltransferase catalyzes the final step of anthocyanin formation in arabidopsis. J. Plant Physiol. 2013, 170, 619-624. [CrossRef] [PubMed]

13. Roepke, J.; Gordon, H.O.W.; Neil, K.J.A.; Gidda, S.; Mullen, R.T.; Freixas Coutin, J.A.; Bray-Stone, D.; Bozzo, G.G. An apoplastic $\beta$-glucosidase is essential for the degradation of flavonol 3-O- $\beta$-glucoside-7-O- $\alpha$-rhamnosides in arabidopsis. Plant Cell Physiol. 2017, 58, 1030-1047. [CrossRef] [PubMed]

14. Barth, C.; Jander, G. Arabidopsis myrosinases tgg1 and tgg2 have redundant function in glucosinolate breakdown and insect defense. Plant J. 2006, 46, 549-562. [CrossRef] [PubMed]

15. Wittstock, U.; Burow, M. Glucosinolate breakdown in arabidopsis: Mechanism, regulation and biological significance. Arabidopsis Book 2010, 8, e0134. [CrossRef] [PubMed]

16. Zhou, C.; Tokuhisa, J.G.; Bevan, D.R.; Esen, A. Properties of $\beta$-thioglucoside hydrolases (tgg1 and tgg2) from leaves of arabidopsis thaliana. Plant Sci. 2012, 191-192, 82-92. [CrossRef] [PubMed]

17. Lee, K.H.; Hai, L.P.; Kim, H.Y.; Sang, M.C.; Fan, J.; Hartung, W.; Hwang, I.; Kwak, J.M.; Lee, I.J.; Hwang, I. Activation of glucosidase via stress-induced polymerization rapidly increases active pools of abscisic acid. Cell 2006, 126, 1109-1120. [CrossRef] [PubMed]

18. Xu, Z.Y.; Lee, K.H.; Dong, T.; Jeong, J.C.; Jin, J.B.; Kanno, Y.; Kim, D.H.; Kim, S.Y.; Seo, M.; Bressan, R.A.; et al. A vacuolar beta-glucosidase homolog that possesses glucose-conjugated abscisic acid hydrolyzing activity plays an important role in osmotic stress responses in arabidopsis. Plant Cell 2012, 24, 2184-2199. [CrossRef] [PubMed]

19. Costet, L.; Fritig, B.; Kauffmann, S. Scopoletin expression in elicitor-treated and tobacco mosaic virus-infected tobacco plants. Physiol. Plant 2002, 115, 228-235. [CrossRef] [PubMed]

20. Fabienne, B.; Patrice, D.R.; Serge, K. Molecular cloning and biological activity of alpha-, beta-, and gamma-megaspermin, three elicitins secreted by phytophthora megasperma h20. Plant Physiol. 2003, 131, 155-166.

21. Hino, F.; Okazaki, M.; Miura, Y. Effect of 2,4-dichlorophenoxyacetic acid on glucosylation of scopoletin to scopolin in tobacco tissue culture. Plant Physiol. 1982, 69, 810-813. [CrossRef] [PubMed]

22. Ahn, Y.O.; Shimizu, B.; Sakata, K.; Gantulga, D.; Zhou, Z.; Bevan, D.R.; Esen, A. Scopolin hydrolyzing beta-glucosidases in roots of arabidopsis. Plant Cell Physiol. 2010, 51, 132. [CrossRef] [PubMed]

23. Chapelle, A.; Morreel, K.; Vanholme, R.; Le-Bris, P.; Morin, H.; Lapierre, C.; Boerjan, W.; Jouanin, L.; Demont-Caulet, N. Impact of the absence of stem-specific beta-glucosidases on lignin and monolignols. Plant Physiol. 2012, 160, 1204-1217. [CrossRef] [PubMed]

24. Zamioudis, C.; Hanson, J.; Pieterse, C.M. Beta-glucosidase bglu42 is a myb72-dependent key regulator of rhizobacteria-induced systemic resistance and modulates iron deficiency responses in arabidopsis roots. New Phytol. 2014, 204, 368-379. [CrossRef] [PubMed]

25. Piffanelli, P.; Ross, J.H.E.; Murphy, D.J. Biogenesis and function of the lipidic structures of pollen grains. Sex. Plant Reprod. 1998, 11, 65-80. [CrossRef]

26. Jiang, J.; Zhang, Z.; Cao, J. Pollen wall development: The associated enzymes and metabolic pathways. Plant Biol. 2013, 15, 249-263. [CrossRef]

27. Minic, Z.; Jouanin, L. Plant glycoside hydrolases involved in cell wall polysaccharide degradation. Plant Physiol. Biochem. 2006, 44, 435-449. [CrossRef] [PubMed]

28. Zhang, D.S.; Liang, W.Q.; Yuan, Z.; Li, N.; Shi, J.; Wang, J.; Liu, Y.M.; Yu, W.J.; Zhang, D.B. Tapetum degeneration retardation is critical for aliphatic metabolism and gene regulation during rice pollen development. Mol. Plant 2008, 1, 599-610. [CrossRef] [PubMed]

29. Dong, X.; Feng, H.; Xu, M.; Lee, J.; Kim, Y.K.; Lim, Y.P.; Piao, Z.; Park, Y.D.; Ma, H.; Hur, Y. Comprehensive analysis of genic male sterility-related genes in brassica rapa using a newly developed br300k oligomeric chip. PLoS ONE 2013, 8, e72178. [CrossRef]

30. Singh, A.K.; Sharma, V.; Pal, A.K.; Acharya, V.; Ahuja, P.S. Genome-wide organization and expression profiling of the nac transcription factor family in potato (Solanum tuberosum L.). DNA Res. 2013, 20, 403-423. [CrossRef] [PubMed] 
31. Larkin, M.A.; Blackshields, G.; Brown, N.P.; Chenna, R.; McGettigan, P.A.; McWilliam, H.; Valentin, F.; Wallace, I.M.; Wilm, A.; Lopez, R.; et al. Clustal w and clustal x version 2.0. Bioinformatics 2007, 23, 2947-2948. [CrossRef] [PubMed]

32. Thorlby, G.; Fourrier, N.; Warren, G. The sensitive to freezing2 gene, required for freezing tolerance in arabidopsis thaliana, encodes a beta-glucosidase. Plant Cell 2004, 16, 2192-2203. [CrossRef] [PubMed]

33. Hu, B.; Jin, J.; Guo, A.Y.; Zhang, H.; Luo, J.; Gao, G. Gsds 2.0: An upgraded gene feature visualization server. Bioinformatics 2015, 31, 1296-1297. [CrossRef]

34. Ng, C.Y.; Wickneswari, R.; Choong, C.Y. Identification of floral genes for sex determination in Calamus palustris griff. By using suppression subtractive hybridization. Genet. Mol. Res. 2014, 13, 6037-6049. [CrossRef]

35. Zhang, L.; Cai, X.; Wu, J.; Liu, M.; Grob, S.; Cheng, F.; Liang, J.; Cai, C.; Liu, Z.; Liu, B.; et al. Improved Brassica rapa reference genome by single-molecule sequencing and chromosome conformation capture technologies. Hortic. Res. 2018, 5, 50. [CrossRef]

36. Rubinelli, P.; Hu, Y.; Ma, H. Identification, sequence analysis and expression studies of novel anther-specific genes of Arabidopsis thaliana. Plant Mol. Biol. 1998, 37, 607-619. [CrossRef]

37. Winter, D.; Vinegar, B.; Nahal, H.; Ammar, R.; Wilson, G.V.; Provart, N.J. An "electronic fluorescent pictograph" browser for exploring and analyzing large-scale biological data sets. PLoS ONE 2007, 2, e718. [CrossRef] [PubMed]

38. Smyth, D.R.; Bowman, J.L.; Meyerowitz, E.M. Early flower development in arabidopsis. Plant Cell 1990, 2, 755-767. [CrossRef] [PubMed]

39. Ma, H. Molecular genetic analyses of microsporogenesis and microgametogenesis in flowering plants. Annu. Rev. Plant Biol. 2005, 56, 393-434. [CrossRef] [PubMed]

40. Wang, P.; Liu, H.; Hua, H.; Wang, L.; Song, C.-P. A vacuole localized $\beta$-glucosidase contributes to drought tolerance in arabidopsis. Chin. Sci. Bull. 2011, 56, 3538-3546. [CrossRef]

41. Wang, X.; Wang, H.; Wang, J.; Sun, R.; Wu, J.; Liu, S.; Bai, Y.; Mun, J.H.; Bancroft, I.; Cheng, F.; et al. The genome of the mesopolyploid crop species Brassica rapa. Nat. Genet. 2011, 43, 1035-1039. [CrossRef] [PubMed]

42. Nuruzzaman, M.; Manimekalai, R.; Sharoni, A.M.; Satoh, K.; Kondoh, H.; Ooka, H.; Kikuchi, S. Genome-wide analysis of nac transcription factor family in rice. Gene 2010, 465, 30-44. [CrossRef] [PubMed]

43. Fourrier, N.; Bedard, J.; Lopez-Juez, E.; Barbrook, A.; Bowyer, J.; Jarvis, P.; Warren, G.; Thorlby, G. A role for sensitive to freezing2 in protecting chloroplasts against freeze-induced damage in arabidopsis. Plant J. 2008, 55, 734-745. [CrossRef] [PubMed]

44. Moellering, E.R.; Bagyalakshmi, M.; Christoph, B. Freezing tolerance in plants requires lipid remodeling at the outer chloroplast membrane. Science 2010, 330, 226-228. [CrossRef] [PubMed]

45. Andreasson, E.; Bolt Jorgensen, L.; Hoglund, A.S.; Rask, L.; Meijer, J. Different myrosinase and idioblast distribution in arabidopsis and brassica napus. Plant Physiol. 2001, 127, 1750-1763. [CrossRef] [PubMed]

46. Talalay, P.; Fahey, J.W. Phytochemicals from cruciferous plants protect against cancer by modulating carcinogen metabolism. J. Nutr. 2001, 131, 3027S-3033S. [CrossRef]

47. Jeffery, E.H.; Araya, M. Physiological effects of broccoli consumption. Phytochem. Rev. 2009, 8, $283-298$. [CrossRef]

48. Borpatragohain, P.; Rose, T.J.; King, G.J. Fire and brimstone: Molecular interactions between sulfur and glucosinolate biosynthesis in model and crop brassicaceae. Front. Plant Sci. 2016, 7, 1735. [CrossRef] [PubMed]

49. Baskar, V.; Gururani, M.A.; Yu, J.W.; Park, S.W. Engineering glucosinolates in plants: Current knowledge and potential uses. Appl. Biochem. Biotechnol. 2012, 168, 1694-1717. [CrossRef] [PubMed]

50. Becker, T.M.; Jeffery, E.H.; Juvik, J.A. Proposed method for estimating health-promoting glucosinolates and hydrolysis products in broccoli (Brassica oleracea var. Italica) using relative transcript abundance. J. Agric. Food Chem. 2017, 65, 301-308. [CrossRef] [PubMed]

51. Kai, K.; Shimizu, B.; Mizutani, M.; Watanabe, K.; Sakata, K. Accumulation of coumarins in arabidopsis thaliana. Phytochemistry 2006, 67, 379-386. [CrossRef]

52. Baiya, S.; Mahong, B.; Lee, S.; Jeon, J.S.; Cairns, J.R.K. Demonstration of monolignol $\beta$-glucosidase activity of rice os4bglu14, os4bglu16 and os4bglu18 in arabidopsis thaliana bglu 45 mutant. Plant Physiol. Biochem. 2018, 127, 223. [CrossRef] [PubMed] 
53. Zhu, J.; Chen, H.; Li, H.; Gao, J.F.; Jiang, H.; Wang, C.; Guan, Y.F.; Yang, Z.N. Defective in tapetal development and function 1 is essential for anther development and tapetal function for microspore maturation in arabidopsis. Plant J. 2008, 55, 266-277. [CrossRef]

54. Eisen, M.B.; Spellman, P.T.; Brown, P.O.; Botstein, D. Correction: Cluster analysis and display of genome-wide expression patterns. Proc. Natl. Acad. Sci. USA 1998, 95, 14863-14868. [CrossRef]

55. Usadel, B.; Obayashi, T.; Mutwil, M.; Giorgi, F.M.; Bassel, G.W.; Tanimoto, M.; Chow, A.; Steinhauser, D.; Persson, S.; Provart, N.J. Co-expression tools for plant biology: Opportunities for hypothesis generation and caveats. Plant Cell Environ. 2009, 32, 1633-1651. [CrossRef]

56. Eisen, M.B.; Spellman, P.T.; Brown, P.O.; Botstein, D. Cluster analysis and display of genome-wide expression patterns. Proc. Natl. Acad. Sci. USA 1998, 95, 14863-14868. [CrossRef] [PubMed]

57. Gillis, J.; Pavlidis, P. "Guilt by association" is the exception rather than the rule in gene networks. PLoS Comput. Biol. 2012, 8, e1002444. [CrossRef] [PubMed]

58. van Dam, S.; Vosa, U.; van der Graaf, A.; Franke, L.; de Magalhaes, J.P. Gene co-expression analysis for functional classification and gene-disease predictions. Brief Bioinf. 2018, 19, 575-592. [CrossRef]

59. Zheng, J.; He, C.; Qin, Y.; Lin, G.; Park, W.D.; Sun, M.; Li, J.; Lu, X.; Zhang, C.; Yeh, C.T.; et al. Co-expression analysis aids in the identification of genes in the cuticular wax pathway in maize. Plant J. 2019, 97, 530-542. [CrossRef] [PubMed]

60. Clough, S.J.; Bent, A.F. Floral dip: A simplified method for agrobacterium-mediated transformation of arabidopsis thaliana. Plant J. 1998, 16, 735-743. [CrossRef]

61. Peterson, R.; Slovin, J.P.; Chen, C. A simplified method for differential staining of aborted and non-aborted pollen grains. Int. J. Plant Biol. 2010, 1, e13. [CrossRef]

62. El-Gebali, S.; Mistry, J.; Bateman, A.; Eddy, S.R.; Luciani, A.; Potter, S.C.; Qureshi, M.; Richardson, L.J.; Salazar, G.A.; Smart, A.; et al. The pfam protein families database in 2019. Nucleic Acids Res. 2018, 47, D427-D432. [CrossRef] [PubMed]

63. Tamura, K.; Stecher, G.; Peterson, D.; Filipski, A.; Kumar, S. Mega6: Molecular evolutionary genetics analysis version 6.0. Mol. Biol. Evol. 2013, 30, 2725-2729. [CrossRef] [PubMed]

64. Wang, Y.; Tang, H.; Debarry, J.D.; Tan, X.; Li, J.; Wang, X.; Lee, T.H.; Jin, H.; Marler, B.; Guo, H.; et al. Mcscanx: A toolkit for detection and evolutionary analysis of gene synteny and collinearity. Nucleic Acids Res. 2012, 40, e49. [CrossRef] [PubMed]

65. Toufighi, K.; Brady, S.M.; Austin, R.; Ly, E.; Provart, N.J. The botany array resource: E-northerns, expression angling, and promoter analyses. Plant J. 2005, 43, 153-163. [CrossRef]

66. Du, Z.; Zhou, X.; Ling, Y.; Zhang, Z.; Su, Z. Agrigo: A go analysis toolkit for the agricultural community. Nucleic Acids Res. 2010, 38, W64-W70. [CrossRef] [PubMed]

(C) 2019 by the authors. Licensee MDPI, Basel, Switzerland. This article is an open access article distributed under the terms and conditions of the Creative Commons Attribution (CC BY) license (http:/ / creativecommons.org/licenses/by/4.0/). 